Artículo científico

(Original paper)

\title{
HORMIGAS (HYMENOPTERA: FORMICIDAE) DEL ESTADO DE SINALOA, MÉXICO
}

\section{ANTS (HYMENOPTERA: FORMICIDAE) FROM THE STATE OF SINALOA, MEXICO}

\author{
MAdAi ROSAS-MEJÍA ${ }^{1}$, Miguel VÁSQUEZ-BOLAÑOS ${ }^{2}$, GRISELda GAONA GARCÍA ${ }^{1}$, VenANCio \\ VANOYE-ELIGIO ${ }^{1 *}$ \\ ${ }^{1}$ Universidad Autónoma de Tamaulipas, Instituto de Ecología Aplicada. División del Golfo 356, Col. Libertad, Cd. Victoria, \\ Tamaulipas, C.P. 87019, México.<marosas@docentes.uat.edu.mx>; <ggaona@docentes.uat.edu.mx>; \\ <vvanoye@docentes.uat.edu.mx> \\ ${ }^{2}$ Entomología, Centro de Estudios en Zoología, Departamento de Botánica y Zoología, Centro Universitario de Ciencias \\ Biológicas y Agropecuarias, Universidad de Guadalajara, Ramón Padilla Sánchez \# 2100, Las Agujas, Zapopan, Jalisco, C.P. \\ 45220, México.<mvb14145@hotmail.com> \\ *Autor corresponsal: <vvanoye@docentes.uat.edu.mx>
}

Recibido: 25/10/2019; aceptado: 28/04/2020; publicado en línea: 16/06/2020

Editor responsable: Magdalena Cruz

Rosas-Mejía, M., Vásquez-Bolaños, M., Gaona García, G., Vanoye-Eligio, V. (2020) Hormigas (Hymenoptera: Formicidae) del estado de Sinaloa, México. Acta Zoológica Mexicana (nueva serie), $36,1-17$. https://doi.org/10.21829/azm.2020.3612250

RESUMEN. En México se ha realizado escaso trabajo taxonómico de las hormigas, particularmente en los estados del noroeste, como Sinaloa. Este estudio tuvo como objetivo revisar la información disponible para los registros de especies de hormigas en el estado de Sinaloa, México. Se muestrearon 11 sitios que comprendieron el $61 \%$ de los municipios de Sinaloa y se realizó una revisión exhaustiva de artículos, páginas web y todo tipo de información especializada disponible sobre Formicidae. Se encontraron un total de 91 especies pertenecientes a 35 géneros. La revisión de la información reveló que el $85.7 \%$ de los registros de hormigas involucraban zonas rurales y urbanas, así como bordes de carreteras. Las selvas bajas caducifolias representaron el $7.61 \%$, seguidos por los bosques espinosos y los mezquitales con $2.85 \%$ y $1 \%$, respectivamente. Se reconoce un total de ocho especies introducidas para Sinaloa. Este es el primer estudio para documentar el conocimiento de Formicidae en Sinaloa. Se sugiere más investigación taxonómica para explorar la diversidad y hábitats relacionados.

Palabras clave: lista comentada; norte de México; taxonomía; especies introducidas

Rosas-Mejía, M., Vásquez-Bolaños, M., Gaona García, G., Vanoye-Eligio, V. (2020) Ants (Hymenoptera: Formicidae) from the State of Sinaloa, Mexico. Acta Zoológica Mexicana (nueva serie), 36, 1-17.

https://doi.org/10.21829/azm.2020.3612250

ABSTRACT. Little taxonomic work of ants has been conducted in Mexico, particularly in the north-west states, such as Sinaloa. This study aimed to review the available information for records of ant species in the state of Sinaloa, Mexico. We sampled 11 sites that comprised $61 \%$ of the municipalities of Sinaloa and 
revised articles, web pages and all kinds of available specialized information on Formicidae. A total of 91 species belonging to 35 genera were listed. The reviewed information revealed that $85.7 \%$ of ant records involved rural and urban zones, as well as roadsides. Low-deciduous forests accounted for $7.61 \%$, followed by thorn forests and mesquite fields with $2.85 \%$ and $1 \%$, respectively. A total of eight introduced species for Sinaloa are recognized. This is the first study aimed to document the knowledge of Formicidae in Sinaloa. Further taxonomic research is suggested to explore such diversity and related habitats.

Key words: checklist; north of Mexico; taxonomy; introduced species

\section{INTRODUCCIÓN}

Las hormigas presentan una amplia variedad de gremios tróficos y funcionales, ofrecen diversos servicios ecosistémicos, como el control de plagas en cultivos, la aireación del suelo y remoción de materia orgánica (De Vega et al., 2014; Arenas-Clavijo \& Armbrecht, 2018; Gallardo et al., 2018). A nivel global, se registran más de 16,000 especies pertenecientes a 474 géneros, contenidos en 20 subfamilias (Bolton, 2018). En México se han registrado 973 especies pertenecientes a 94 géneros en 11 subfamilias, lo que representa aproximadamente el 6\% del total mundial (Coronado-Blanco et al., 2013; Ríos-Casanova, 2014; VásquezBolaños, 2015). Sin embargo, el poco trabajo taxonómico realizado y gran parte del territorio aún sin explorar, hacen que no se tenga la certeza del número total de especies en el país (Rojas-Fernández, 2011; Vásquez-Bolaños, 2011). Para el estado de Sinaloa se tienen registrados 23 géneros y apenas 52 especies (Rosas-Mejía et al., 2019), lo cual es un número muy bajo si se compara con lo registrado para otros estados del norte de México como Baja California, Tamaulipas y Sonora con 161, 155 y 135 especies, respectivamente (Alatorre-Bracamontes \& Vásquez-Bolaños, 2010). El objetivo de este trabajo fue actualizar la información de la mirmecofauna para el estado de Sinaloa, así como revisar el estado de conocimiento de las hormigas de Sinaloa.

\section{MATERIALES Y MÉTODOS}

Se revisó exhaustivamente la información publicada sobre las especies de hormigas en el estado de Sinaloa. Las fuentes consultadas incluyeron artículos y notas científicas que mencionan al menos una especie registrada en Sinaloa, México. Así como, páginas en internet especializadas tales como AntWeb (AntWeb, 2018), Antmaps (Janicki et al., 2016) y AntCat (Bolton, 2018), en donde se tuvo acceso a bases de datos. A continuación, se entrega la información de las colecciones y museos para el caso de especímenes resguardados:

[ANIC]: Australian National Insect Collection, [CASC]: California Academy of Science, Michelbacher, [FMNH]: Field Museum of Natural History, Chicago, IL, USA, [LACM]: Los Angeles County Museum of Natural History,[MIEA]: Colección Entomológica del Instituto de Ecología Aplicada de la Universidad Autónoma de Tamaulipas, Ciudad Victoria Tamaulipas, [MWP]: Personal Collection of Mackay, W. P., [PSWC]: Philip S. Ward Collection, [RAJC]: Robert A. Johnson Collection, [USNM]: United States National Museum of Natural History Washington D.C., [CZUG]: Colección Entomológica de la Universidad de Guadalajara. Adicionalmente, se incluyeron los registros de 15 especies de hormigas recolectadas entre junio y septiembre del 2014, y entre febrero y abril del 2015 en tres localidades del norte del estado entre los municipios de Ahome y Guasave: Tajito ( $25^{\circ} 39^{\prime} 27.4^{\prime \prime} \mathrm{N}, 108^{\circ} 38^{\prime} 04.8^{\prime \prime} \mathrm{O}$ ), Juan José Ríos ( $\left.25^{\circ} 45^{\prime} 23.7^{\prime \prime} \mathrm{N}, 108^{\circ} 49^{\prime} 13.0^{\prime \prime} \mathrm{O}\right)$ y Villa de Ahome (25 55' 10.6" N, 109 10' 27.2" O). El material se encuentra depositado en la Colección Entomológica del Instituto de Ecología Aplicada de la Universidad Autónoma de Tamaulipas, Ciudad Victoria Tamaulipas (MIEA). 
Se presenta información sobre cada muestra obtenida durante 2014-2015. También se incorporan los datos obtenidos para cada registro en el estado de Sinaloa, citando la fuente de donde se obtuvo la información. Algunos registros de especies no tienen información precisa sobre las localidades donde se realizó el muestreo. El arreglo taxonómico de subfamilias, tribus y géneros sigue al de Vásquez-Bolaños (2015).

\section{RESULTADOS Y DISCUSIÓN}

El número total de especies registradas actualmente para el estado de Sinaloa es de 91 dentro de 35 géneros y siete subfamilias en 11 municipios. Estas cifras representan el $9 \%$ del total del número de especies y $37.3 \%$ de los géneros registrados para México (Ríos-Casanova, 2014; Vásquez-Bolaños, 2015). Lo anterior se puede comparar con lo registrado para otros estados, en Tamaulipas se registran 155 especies (15.93\%) y 53 géneros (54\%), respectivamente (Coronado-Blanco et al., 2013), en Tlaxcala 25 especies de hormigas $(2.56 \%)$ y 12 géneros (12.7\%) (Dubovikoff \& Coronado-Blanco, 2017) y en Veracruz 310 especies (31.8\%) y 72 géneros (76.6\%) (Ríos-Casanova, 2014). Estas diferencias cuantitativas de diversidad en los estados dependen de diversos factores, entre los que destacan el clima, la humedad, la vegetación, el esfuerzo de muestreo y las condiciones biogeográficas (Guzmán-Mendoza et al., 2010). En el norte de México se han realizado estudios donde registran las especies encontradas incluyendo morfoespecies para los estados de Baja California (161), Tamaulipas (155) y Sonora (135) (Alatorre-Bracamontes \& Vásquez-Bolaños, 2010). Al comparar estos datos con los resultados obtenidos para Sinaloa, se puede observar que se obtiene un mayor número de especies en otras entidades del norte, contrastantes con este estado por diversas condiciones climáticas (Cuadro 1). En el caso de Sinaloa con una extensión territorial de 57,365 km² debería esperarse un mayor número de especies de hormigas debido a su ubicación en una zona de transición mexicana de montaña donde se superponen biotas neárticas y neotropicales (Halffter, 1976; MiguezGutiérrez et al., 2013). En el estado se encuentran diferentes ecosistemas tales como el bosque espinoso, bosque de coníferas, bosque de encinos y bosque mesófilo (Vega-Aviña, 2000). Sin embargo, las recolectas de hormigas que se han hecho en Sinaloa se concentraron en zonas perturbadas (Fig. 1), probablemente debido a una mayor accesibilidad en estas zonas. Del material revisado que contenía información de hábitat, el $85.7 \%$ de los registros correspondió a zonas rurales, urbanas y bordes de camino seguido de selva baja caducifolia con el $7.61 \%$. Mientras que un menor porcentaje fue observado en bosque espinoso tropical (3.4\%), bosque de pino-encino (2.85\%) y mezquital (1\%). Este bajo porcentaje de registros se puede atribuir a la transición hacia zonas áridas, donde se presenta un menor número de especies de hormigas en comparación con los ecosistemas tropicales (Rojas-Fernández \& Fragoso, 2000). Referente a las especies, se obtuvo el mayor número en las zonas perturbadas, exceptuando las especies sin información de hábitat (Fig. 2, 3). En cuanto al número de especies por subfamilia, se registró la siguiente información: Myrmicinae (40), Formicinae (15), Dorylinae (11), Pseudomyrmecinae (11), Dolichoderinae (8), Ponerinae (5) y Ectatomminae (1). Este patrón concuerda con lo encontrado a nivel mundial en donde Myrmicinae y Formicinae son las subfamilias con mayor riqueza de especies dentro de los diferentes hábitats ocupados por formícidos. Ambos grupos abarcan una gama extraordinaria de comportamientos de alimentación, hábitos de anidación y estructura de colonias (Hölldobler \& Wilson, 1990; Ward, 2010).

El género con mayor número de especies fue Pseudomyrmex (11), seguido de Pogonomyrmex (7) y Pheidole (7) que representan aproximadamente $11.2 \%$ y $7.7 \%$, respectivamente, del número total de especies en el estado. Esto coincide en parte con lo registrado para el país, sin embargo, Pogonomyrmex no figura como un género numeroso a nivel nacional, lo que contrasta con lo informado para Sinaloa. Lo anterior se puede explicar debido al comportamiento dominante observado en zonas áridas y semiáridas del norte de México (Mackay et al., 1985; Soto-Cárdenas et al., 2019). Esto se puede corroborar con la distribución de cinco de las siete especies del género Pogonomyrmex encontradas para Sinaloa en áreas predominantemente áridas (Gúenard et al., 2017). Para el género Atta, se registran las especies Atta mexicana (Smith, 1858) y Atta cephalotes (Linnaeus, 1758), sin embargo, la presencia de esta última 
requiere de mayor estudio debido a que sólo existe un registro dado por Padilla y Miyazawa (1972). En este trabajo no se obtuvieron datos adicionales para el estado y las entidades vecinas, solo en el estado norteño de Tamaulipas. La distribución para esta especie abarca desde Sudamérica, zonas bajas del sur y sureste de México, así como parte centro (Gúenard et al., 2017).

Cuadro 1. Riqueza de especies en diferentes estados del Norte de México.

\begin{tabular}{ccc}
\hline Estados del norte de México & Riqueza de especies & Fuente \\
\hline Sinaloa & 91 & Derivado del análisis en el presente estudio \\
Baja California & 161 & Alatorre-Bracamontes y Vásquez-Bolaños, 2010 \\
Sonora & 135 & Alatorre-Bracamontes y Vásquez-Bolaños, 2010 \\
Nuevo León & 107 & Alatorre-Bracamontes y Vásquez-Bolaños, 2010 \\
Tamaulipas & 155 & Coronado-Blanco et al., 2013 \\
Chihuahua & 42 & Alatorre-Bracamontes y Vásquez-Bolaños, 2010 \\
Coahuila & 41 & Alatorre-Bracamontes y Vásquez-Bolaños, 2010 \\
Durango & 40 & Soto-Cárdenas et al., 2019 \\
\hline
\end{tabular}

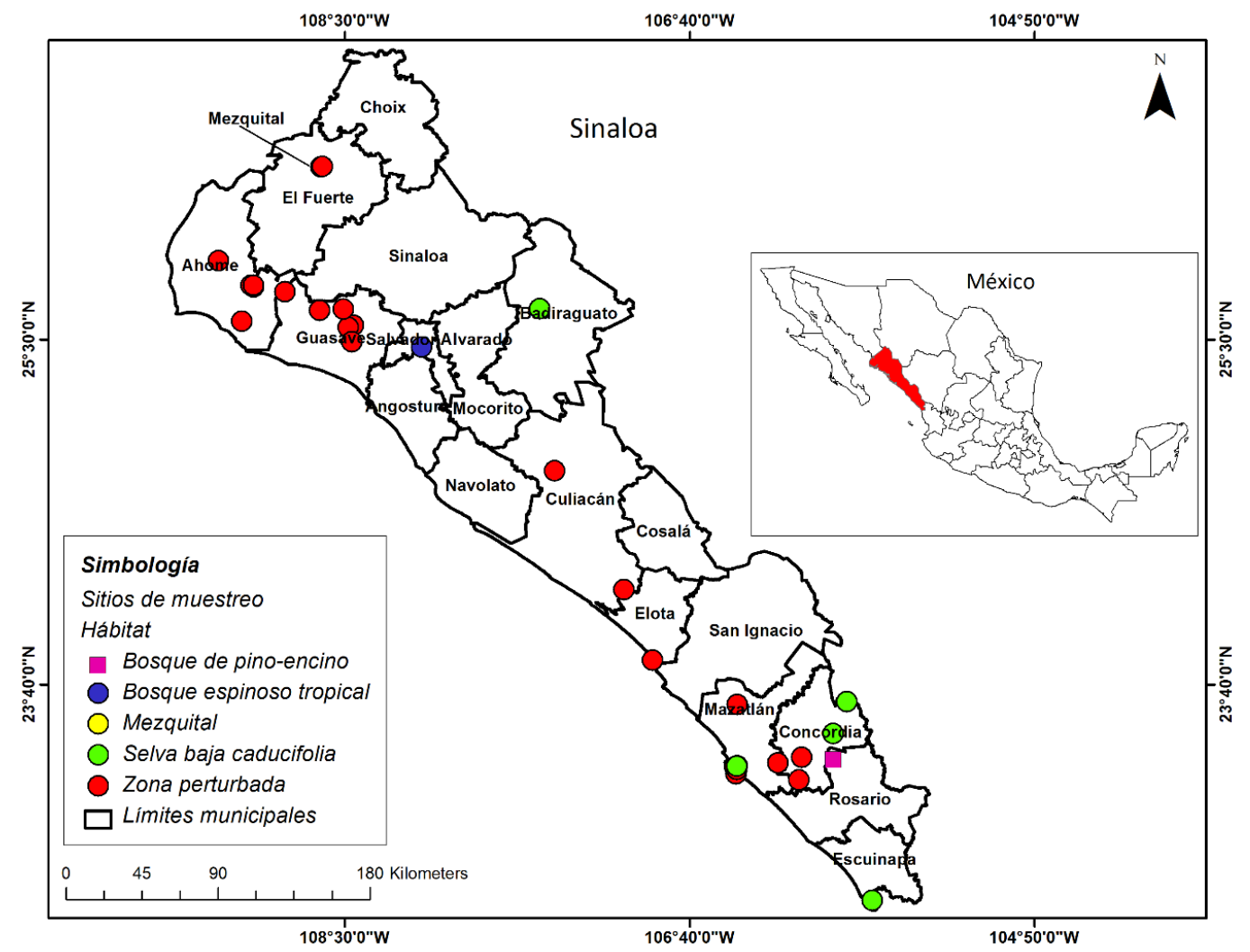

Figura 1. Mapa con indicación de los registros de hormigas por hábitat y municipio para el estado de Sinaloa, México

Mazatlán fue el municipio con mayor número de especies, seguido de Ahome y Guasave, mientras que los municipios de Badiraguato y El Fuerte presentaron menos registros (Fig. 2). Sin embargo, se debe tomar en cuenta que más del $80 \%$ de los registros en este estudio correspondió a zonas rurales y urbanas, y los trabajos sobre mirmecofauna en Sinaloa son escasos y datan de la década de los 60 y 70 . Las hormigas 
introducidas representan aproximadamente el 9\% del total de las especies: Tapinoma melanocephalum (Fabricius, 1793), Monomorium pharaonis (Linnaeus, 1758), Monomorium floricola (Jerdon, 1851), Hypoponera opcaciceps (Mayr, 1887), Tetramorium lanuginosum Mayr, 1870, Tetramorium simillimum (Smith, F., 1851), Paratrechina longicornis (Latreille, 1802) y Anoplolepis gracilipes (Smith, F., 1857). Estas especies se benefician de ambientes antropizados (Wetterer et al., 2019) tales como las zonas perturbadas en donde se realizaron estas colectas. Dentro de este grupo, algunas especies son consideradas como invasoras o plagas, i.e. T. melanocephalum considerada como una plaga doméstica y agrícola capaz de transportar patógenos (Oliveira et al., 2017); Monomorium pharaonis es vector de algunos patógenos humanos (De Castro et al., 2015); Paratrechina longicornis es capaz de desplazar a otras hormigas (Roxo et al., 2010), y A. gracilipes es reportada en la invasión de ecosistemas y causante de daños al medio ambiente (Plentovich et al., 2018). En el estado, no se tienen informes sobre los daños que estas hormigas puedan estar causando, por lo que se detecta la necesidad de estudiar el posible impacto de las hormigas introducidas en Sinaloa. En general, se puede detectar la falta de estudios dirigidos al conocimiento de Formicidae en Sinaloa.

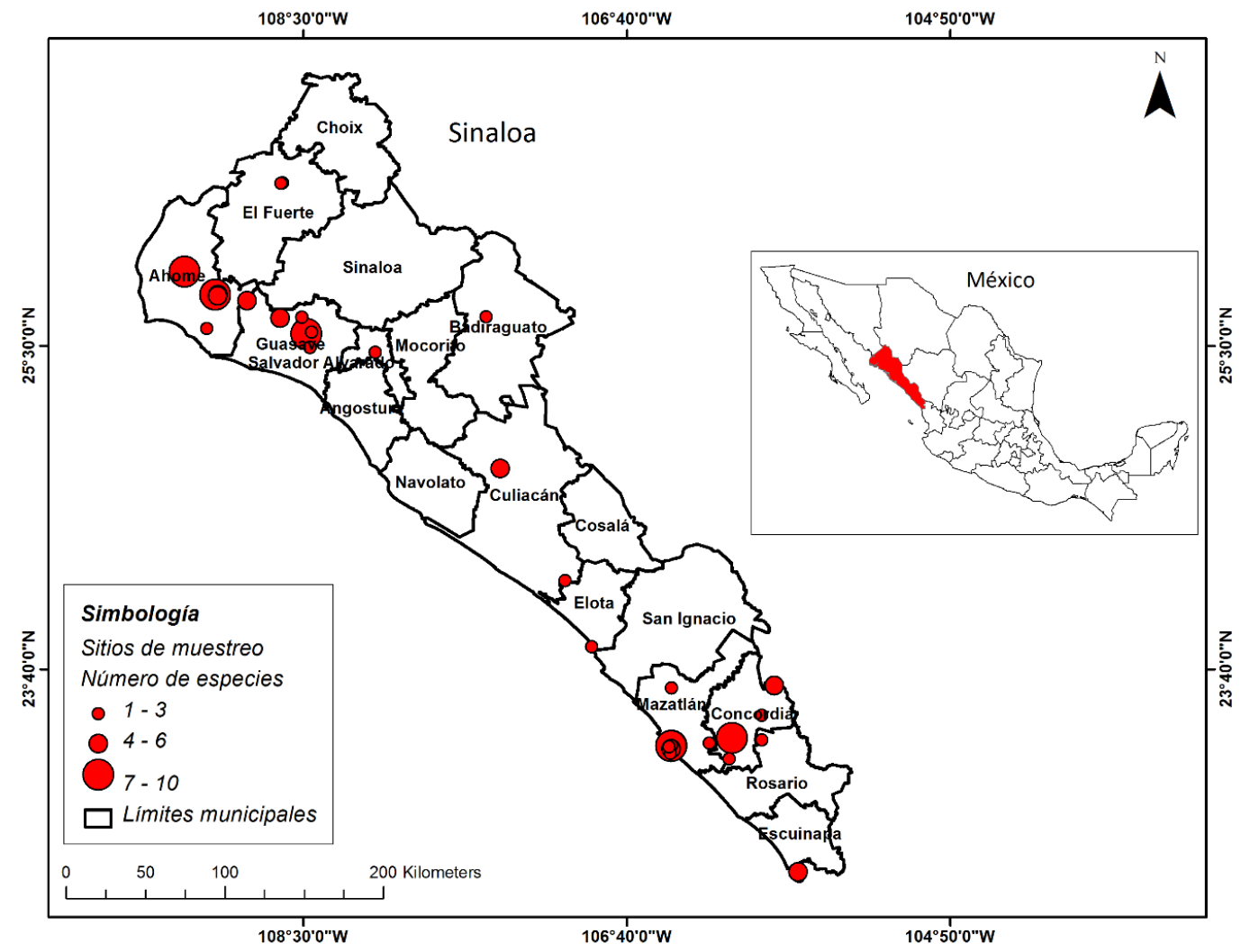

Figura 2. Número de especies por sitio de muestreo en el estado de Sinaloa.

\section{Información sobre hormigas (Hymenoptera: Formicidae) del estado de Sinaloa, México}

Los datos obtenidos sobre cada registro, así como la información recopilada para cada especie reportada para Sinaloa, México se presentan en el Anexo I. 


\title{
Número de especies
}

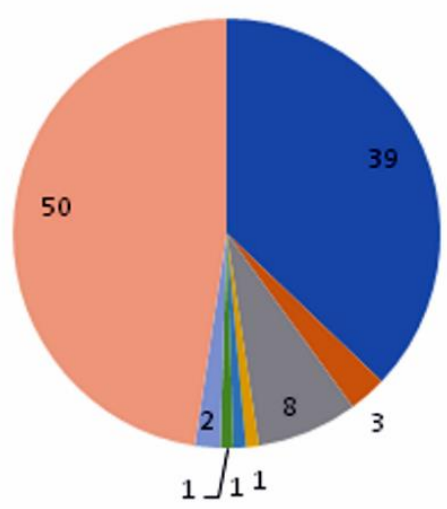

\author{
- Zona perturbada \\ - Bosque de pino-encino \\ - Selvabaja caducifolia \\ - Bosque espinoso tropical \\ Mezquital \\ - Tierras bajas costeras \\ - Acantilado rocoso \\ Inin información
}

Figura 3. Número de especies registradas por tipo de vegetación. Nota: Se anexan las especies que no presentaron información al respecto. La clasificación de la vegetación es de acuerdo con INEGI (2017) y la información original de colecta presentada por AntWeb (2020).

\section{CONCLUSIONES}

El presente trabajo encuentra que Sinaloa tiene un registro de 91 especies de hormigas, de las cuales un alto porcentaje se registraron en zonas perturbadas $(85.7 \%)$. Basado en el número de registros de especies que se han realizado en selva baja caducifolia $(7.61 \%)$, bosque espinoso $(2.85 \%)$ y mezquital (1\%), se sugiere que gran parte de la diversidad de hormigas es desconocida comparada con la información registrada para otros estados del norte de México. En Sinaloa, el número de especies puede ser mayor si el muestreo se extiende a zonas sin explorar cómo en el caso del centro de la entidad. Una opción para futuros trabajos es la obtención de muestras con diversas técnicas como trampas Winkler, pitfall y cebos, en diferentes meses, especialmente después de las lluvias, debido a que, en bosques tropicales, el ciclo de las colonias de hormigas se sincroniza con la precipitación y la temperatura estacional (Kaspari et al., 2001; Nene, 2015).

AgRADECIMIENTOS. Se agradece a Benoit Guénard por el acceso a la información de la base de datos: Global Ant Biodiversity Informatics (GABI).

\section{LITERATURA CITADA}

Alatorre-Bracamontes, C. E., Vásquez-Bolaños, M. (2010) Lista comentada de las hormigas (Hymenoptera: Formicidae) del norte de México. Dugesiana, 17, 9-36. http://dx.doi.org/10.32870/dugesiana.v26i2.7078

AntWeb (2018) All AntWeb, en California Academy of Sciences. Disponible en: https://www.antweb.org/ (última consulta en marzo 2018).

Arenas-Clavijo, A., Armbrecht, I. (2018) Gremios y diversidad de hormigas (Hymenoptera: Formicidae) en tres usos del suelo de un paisaje cafetero del Cauca-Colombia. Revista de Biología Tropical, 66, $48-57$. https://doi.org/10.1673/031.013.2901

Bolton, B. (2018) AntWeb: Bolton World Catalog, en California Academy of Sciences. Disponible en: https://www.antweb.org/project.do?name=worldants (última consulta en marzo 2018). 
Branstetter, M. G. (2013) Revision of the Middle American clade of the ant genus Stenamma westwoodi (Hymenoptera, Formicidae, Myrmicinae). ZooKeys, 295, 1-277.

http://dx.doi.org/10.3897/zookeys.295.4905

Coronado-Blanco, J. M., Dubovikoff, D. A., Ruíz-Cancino, E., Vásquez-Bolaños, M., FloresMaldonado, K. Y., Horta-Vega, J. V. (2013) Formicidae (Hymenoptera) del estado de Tamaulipas, México. CienciaUAT, 7, 12-17.

Cuezzo, F., Guerrero, R. J. (2011) The ant genus Dorymyrmex Mayr (Hymenoptera: Formicidae: Dolichoderinae) in Colombia. Psyche (Cambridge), 2012, 1-24. http://dx.doi.org/10.1155/2012/516058

De Andrade, M. L., Baroni-Urbani, C. (1999) Diversity and adaptation in the ant genus Cephalotes, past and present. Stuttgarter Beiträge zur Naturkunde Serie b, 198, 1-65.

De Castro, M. M., Prezoto, H. H. S., Fernandes, E. F., Bueno, O. C., Prezoto, F. (2015) The ant fauna of hospitals: advancements in public health and research priorities in Brazil. Revista Brasileira de entomología, 59, 77-83. http://dx.doi.org/10.1016/j.rbe.2015.02.011

De Vega, C., Herrera, C. M., Dötterl, S. (2014) Floral volatiles play a key role in specialized ant pollination. Perspectives in Plant Ecology, Evolution and Systematics, 16, 32-42. http://dx.doi.org/10.1016/j.ppees.2013.11.002

Del Toro, I., Vázquez, M., Mackay, W. P., Rojas, P., Zapata-Mata, R. (2009) Hormigas (Hymenoptera: Formicidae) de Tabasco: explorando la diversidad de la mirmecofauna en las selvas tropicales de baja altitud. Dugesiana, 16, 1-14. http://dx.doi.org/10.32870/dugesiana.v16i1.3920

DuBois, M. B. (1986) A revision of the native new world species of the ant genus Monomorium (minimum group) (Hymenoptera: Formicidae). The University of Kansas Science Bulletin, 53, 65-119.

Dubovikoff, D. A., Coronado-Blanco, J. M. A. (2017) Checklist of ants (Hymenoptera: Formicidae) of the state of Tlaxcala, Mexico. Caucasian Entomological Bulletin, 13, 235-241. https://doi.org/10.23885/1814-3326-2017-13-2-235-241

Forel, A. (1899) Biologia Centrali-Americana; or, contributions to the knowledge of the fauna and flora of Mexico and Central America. Botany, Londres, $160 \mathrm{pp}$.

Gallardo, G., Barrionuevo, M. J., Scrocchi, G. (2018) Dieta de la lagartija arenícola Liolaemus laurenti (Sauria: Liolaemidae) en un bioma de desierto de Argentina. Cuadernos de Herpetología, 32, 6. https://doi.org/10.31017/11743

Guénard, B., Weiser, M. D., Gomez, K., Narula, N., Economo, E. P. (2017) The Global Ant Biodiversity Informatics (GABI) database: synthesizing data on ant species geographic distribution. Myrmecological News, 24, 83-89. https://doi.org/10.25849/myrmecol.news_024:083

Guzmán-Mendoza, R., Castaño-Meneses, G., Herrera-Fuentes, M. D. C. (2010) Variación espacial y temporal de la diversidad de hormigas en el Jardín Botánico del valle de Zapotitlán de las Salinas, Puebla. Revista mexicana de biodiversidad, 81, 427-435.

http://dx.doi.org/10.22201/ib.20078706e.2010.002.233

Halffter, G. (1976) Distribución de los insectos en la Zona de Transición Mexicana: relaciones con la entomofauna de Norteamérica. Folia Entomológica Mexicana, 35, 1-64.

Hölldobler, B., Wilson, E. O. (1990) The ants. Harvard University Press.

INEGI (2017) INEGI presenta la Carta de Uso del Suelo y Vegetación Serie 6. Disponible en: http://www.beta.inegi.org.mx/contenidos/saladeprensa/boletines/2017/especiales/especiales2017_ 12_01.pdf (última consulta en marzo 2018).

Janicki, J. H., Narula, N., Ziegler, M., Guénard, B., Economo, E. P. (2016) Visualizing and interacting with large-volume biodiversity data using client-server web mapping applications: The design and implementation of antmaps.org. Ecological Informatics, 32, 185-193. http://dx.doi.org/10.1016/j.ecoinf.2016.02.006 1574-9541/ 
Kaspari, M., Pickering, J., Longino, J. T., Windsor, D. (2001) The phenology of a Neotropical ant assemblage: evidence for continuous and overlapping reproduction. Behavioral Ecology and Sociobiology, 50, 382-390. https://doi.org/10.1007/s002650100378

Kempf, W. W. (1972) Catálogo abreviado das formigas da regiâo neotropical (Hymenoptera: Formicidae). Studia Entomologica, 95, 1-345.

Landero-Torres, I., Murguía-Gonzáles, J., Galindo-Tovar, M. E., Leyva-Ovalle, O. R., Presa-Parra, E., Quiroz-Robledo, L. N., Valenzuela-González, J. E., García-Martínez, M. A. (2015) New records of ants species (Hymenoptera: Formicidae) for Tlaxcala, Mexico. Revista Colombiana de Entomología, 41, 275-277. http://dx.doi.org/10.3958/059.038.0411

Lattke, J. E. (1995) Revision of the ant genus Gnamptogenys in the New World (Hymenoptera: Formicidae). Journal of Hymenoptera Research, 4, 137-193.

Longino, J. T. (2003) The Crematogaster (Hymenoptera: Formicidae) of Costa Rica. Zootaxa, 151, 1-150. http://dx.doi.org/10.11646/zootaxa.151.1.1

Mackay, W. P., Mackay, E. E., Pérez Domínguez, J. F., Sánchez, L. V., Vielma-Orozco, P. (1985) Las hormigas del estado de Chihuahua México: el género Pogonomyrmex (Hymenoptera: Formicidae). Sociobiology, 11, 39-54.

Mackay, W. P., Mackay, E. E. (2002) The ants of New Mexico (Hymenoptera: Formicidae). The Edwin Mellen Press, New York, 408 pp.

Miguez-Gutiérrez, A., Castillo, J., Márquez, J., Goyenechea, I. (2013) Biogeografía de la Zona de Transición Mexicana con base en un análisis de árboles reconciliados. Revista Mexicana de Biodiversidad, 84, 215-224. http://dx.doi.org/10.7550/rmb.32119

Morgan, C., Mackay, W. P. (2017) The North America acrobat ants of the hyperdiverse genus Crematogaster. Lambert Academic Publishing, Mauritius, 540 pp.

Oliveira, B. R., De, S. L., Soares, R. C., Nascimento, T. C., Madureira, M. S., Fortuna, J. L. (2017) Ants as vectors of bacteria in hospital environments. Research Journal of Microbiology, 7, 1-7. http://dx.doi.org/10.5923/j.microbiology.20170701.01

Nene, W. A., Rwegasira, G. M., Nielsen, M. G., Mwatawala, M., Offenberg, J. (2016) Nuptial flights behavior of the African weaver ant, Oecophylla longinoda Latreille (Hymenoptera: Formicidae) and weather factors triggering flights. Insectes sociaux, 63, 243-248. https://doi.org/10.1007/s00040-015-0456-9

Padilla, R. C., Miyazawa, E. E. (1972) Distribución geográfica de las especies de hormigas arrieras existentes en la República Mexicana. Fitofilo, 67, 35-36.

Plentovich, S., Russell, T., Fejeran, C. C. (2018) Yellow crazy ants (Anoplolepis gracilipes) reduce numbers and impede development of a burrow-nesting seabird. Biological invasions, 20, 77-86. http://dx.doi.org/10.1007/s10530-017-1516-z

Ríos-Casanova, L. (2014) Biodiversidad de hormigas en México. Revista Mexicana de Biodiversidad, 85, 392-398.

http://dx.doi.org/10.7550/rmb.32519

Rojas-Fernández, P. (1996) Formicidae (Hymenoptera). Pp: 483-500. In: J. B. A. Llorente, N. A. García, E. S. González (Eds.). Biodiversidad, Taxonomía y Biogeografía de Artrópodos de México: Hacia una síntesis de su conocimiento. Instituto de Biología, UNAM, México, D.F.

Rojas-Fernández, P. (2011) Hormigas (Insecta: Hymenoptera: Formicidae). Pp: 431-439. In: A. Cruz Aragón (Ed.). Diversidad biológica de Veracruz volumen invertebrados. CONABIO-Gobierno del Estado, Veracruz, México.

Rojas-Fernández, P., Fragoso, C. (2000) Composition, diversity, and distribution of Chihuahuan Desert ant community (Mapimí, Mexico). Journal of Arid Environments, 44, 213-227.

http://dx.doi.org/10.1006/jare.1999.0583 
Rosas-Mejía, M., Vásquez-Bolaños, M., Gaona-García, G., Vanoye-Eligio, V. (2019) Nuevos registros de especies de hormigas para el estado de Sinaloa, México. Southwestern Entomologist, 44, 551554. https://doi.org/10.3958/059.044.0221

Roxo, E., Campos, A. E. C., Alves, M. P., Couceiro, A. P. M. R., Harakava, R., Ikuno, A. A., Augusto, M. (2010) Ants' role (Hymenoptera: Formicidae) as potential vectors of mycobacteria dispersion. Arquivos do Instituto Biológico (São Paulo), 77, 359-362.

Schmidt, J. O., Snelling, R. R. (1986) Pogonomyrmex occidentalis, an addition to the ant fauna of Mexico, with notes on other species of harvester ants from Mexico (Hymenoptera: Formicidae). The Southwestern Naturalist, 31, 395-396.

Smith, M. R. (1943) Pheidole (Macropheidole) rhea Wheeler, a valid species (Hymenoptera: Formicidae). Proceedings of the Entomological Society of Washington, 45, 5-9.

Snelling, R. R. (1968) Taxonomic notes on some cephalotine ants (Hymenoptera: Formicidae). Contributions in Science, 132, 1-10.

Snelling, R. R., Longino, J. T. (1992) Revisionary notes on the fungus-growing ants of the genus Cyphomyrmex, rimosus group (Hymenoptera: Formicidae: Attini). Pp: 479-494. In: D. Quintero, A. Aiello (Eds.). Insects of Panama and Mesoamerica: selected studies. Oxford University Press, Oxford.

Soto-Cárdenas, M. A., Vásquez-Bolaños, M., Gutiérrez-García, C., Correa-Ramírez, M. M., TorresRicario, R., González-Güereca, M. C., Chairez-Hernández, I. (2019) Diversidad de Hormigas en Ecorregiones de Durango, México. Southwestern Entomologist, 44, 539-550. https://doi.org/10.3958/059.044.0220

Vásquez-Bolaños, M. (2011) Lista de especies de hormigas (Hymenoptera: Formicidae) para México. Dugesiana, 18, 95-133. http://dx.doi.org/10.32870/dugesiana.v18i1.3995

Vásquez-Bolaños, M. (2015) Taxonomía de Formicidae (Hymenoptera) para México. Métodos en Ecología y Sistemática, 10, 1-53.

Vega-Aviña, R. (2000) Catálogo y base de datos preliminar de la flora de Sinaloa. Facultad de Agronomía, Universidad Autónoma de Sinaloa. Base de datos, del proyecto L057, en Sistema Nacional de Información sobre Biodiversidad-Comisión Nacional para el Conocimiento y Uso de la Biodiversidad. Disponible en: http://www.conabio.gob.mx/institucion/proyectos/resultados/Conabio_Contenido\%20L057.pdf. (última consulta en marzo 2018).

Ward, P. S. (1989) Systematic studies on Pseudomyrmecine ants: Revision of the Pseudomyrmex oculatus and $P$. subtilissimus species groups, with taxonomic comments on other species. Quaestiones Entomologicae, 25, 393-468. https://doi.org/10.5281/zenodo.24960

Ward, P. S. (1993) Systematic studies on Pseudomyrmex acacia ants (Hymenoptera: Formicidae: Pseudomyrmecinae). Journal of Hymenoptera Research, 2, 117-168. https://doi.org/10.5281/zenodo.10150

Ward, P. S. (2010) Taxonomy, Phylogenetics, and Evolution. Pp: 3-17. In: L. Lach, C. L. Parr, K. L. Abbott (Eds.). Ant Ecology. Oxford University Press, New York.

Watkins, J. F. (1982) The army ants of Mexico (Hymenoptera: Formicidae: Ecitoninae). Journal of the Kansas Entomological Society, 55, 197-247. https://doi.org/10.2307/25084279

Wetterer, J. K., Lubertazzi, D., Wilson, E. O. (2019) Ants of Grenada (Hymenoptera, Formicidae). Bulletin of the Museum of Comparative Zoology, 162, 309-346. https://doi.org/10.3099/0027-4100-162.5.263

Wilson, E. O. (2003) Pheidole in the New World: a dominant, hyperdiverse ant genus. Harvard University Press, Cambridge, Massachusetts, 794 pp. 
Anexo I. Especies de Formicidae (Hymenoptera) registradas para el estado de Sinaloa (según varios autoresver información de museos en materiales y métodos). Los registros presentados por los autores se indican con un asterisco $(*)$.

\begin{tabular}{|c|c|c|c|c|c|c|c|}
\hline Especie & Municipio & Coordenadas & $\begin{array}{l}\text { Altitud } \\
(\mathrm{msnm})\end{array}$ & $\begin{array}{l}\text { Fecha de } \\
\text { colecta }\end{array}$ & Colectores & Colección & $\begin{array}{l}\text { Fuente de } \\
\text { información }\end{array}$ \\
\hline \multicolumn{8}{|c|}{$\begin{array}{l}\text { SUBFAMILIA DOLICHODERINAE Forel, } 1878 \\
\text { TRIBU: Leptomyrmecini Emery, } 1913\end{array}$} \\
\hline $\begin{array}{l}\text { Azteca alfari } \\
\text { Emery, } 1893\end{array}$ & Guasave & $\begin{array}{l}25^{\circ} 39^{\prime} 27.4^{\prime \prime} \mathrm{N} \\
108^{\circ} 38^{\prime} 04.8^{\prime \prime} \mathrm{O}\end{array}$ & 15 & 06-mar-14 & $\begin{array}{l}\text { Rosas-Mejía, } \\
\text { M. }\end{array}$ & MIEA & $*$ \\
\hline Azteca alfari & Concordia & $\begin{array}{l}23^{\circ} 24^{\prime} 36^{\prime \prime} \mathrm{N} \\
105^{\circ} 54^{\prime} 6.0114^{\prime \prime} \mathrm{O}\end{array}$ & 920 & 15-ene-07 & Ward, P. S. & USNM & AntWeb, 2018 \\
\hline $\begin{array}{l}\text { Dorymyrmex } \\
\text { bicolor Wheeler, } \\
1906\end{array}$ & Ahome & & & & & LACM & $\begin{array}{l}\text { Cuezzo y } \\
\text { Guerrero, } 2011\end{array}$ \\
\hline $\begin{array}{l}\text { Dorymyrmex } \\
\text { bicolor }\end{array}$ & Ahome & $\begin{array}{l}25^{\circ} 47^{\prime} 23 " \mathrm{~N} \\
109^{\circ} 0^{\prime} 1 " \mathrm{O}\end{array}$ & 13 & 06-ago-10 & $\begin{array}{l}\text { Vásquez- } \\
\text { Bolaños, M. }\end{array}$ & CZUG & $*$ \\
\hline $\begin{array}{l}\text { Dorymyrmex } \\
\text { bicolor }\end{array}$ & El Fuerte & $\begin{array}{l}26^{\circ} 25^{\prime} 19^{\prime \prime} \\
\mathrm{N}, 108^{\circ} 37^{\prime} 15^{\prime \prime} \mathrm{O}\end{array}$ & 86 & $05-a b r-15$ & $\begin{array}{l}\text { Vásquez- } \\
\text { Bolaños, M. }\end{array}$ & CZUG & $*$ \\
\hline $\begin{array}{l}\text { Dorymyrmex } \\
\text { insanus } \\
\text { (Buckley, 1866) }\end{array}$ & Ahome & $\begin{array}{l}25^{\circ} 55^{\prime} 10.6^{\prime \prime} \mathrm{N} \\
109^{\circ} 10^{\prime} 27.2^{\prime \prime} \mathrm{O}\end{array}$ & 10 & 08-jul-14 & $\begin{array}{l}\text { Rosas-Mejía, } \\
\text { M. }\end{array}$ & MIEA & * \\
\hline $\begin{array}{l}\text { Dorymyrmex } \\
\text { insanus }\end{array}$ & Sinaloa & & & & & & $\begin{array}{l}\text { Rojas-Fernández, } \\
1996\end{array}$ \\
\hline $\begin{array}{l}\text { Forelius keiferi } \\
\text { Wheeler, } 1934\end{array}$ & Guasave & $\begin{array}{l}25^{\circ} 45^{\prime} 23.7^{\prime \prime} \mathrm{N} \\
108^{\circ} 49^{\prime} 13.0^{\prime \prime} \mathrm{O}\end{array}$ & 12 & $05-a b r-15$ & $\begin{array}{l}\text { Rosas-Mejía, } \\
\text { M. }\end{array}$ & MIEA & ( \\
\hline Forelius keiferi & Ahome & $\begin{array}{l}25^{\circ} 47^{\prime} 23^{\prime \prime} \mathrm{N} \\
109^{\circ} 00^{\prime} 1 \mathrm{O}\end{array}$ & 13 & 06-ago-10 & $\begin{array}{l}\text { Vásquez- } \\
\text { Bolaños, M. }\end{array}$ & CZUG & $*$ \\
\hline Forelius keiferi & El Fuerte & $\begin{array}{l}26^{\circ} 25^{\prime} 19^{\prime \prime} \mathrm{N} \\
108^{\circ} 37^{\prime} 15^{\prime \prime} \mathrm{O}\end{array}$ & 86 & $05-a b r-15$ & $\begin{array}{l}\text { Vásquez- } \\
\text { Bolaños, M. }\end{array}$ & CZUG & $*$ \\
\hline Forelius keiferi & Guasave & $\begin{array}{l}25^{\circ} 34^{\prime} 5^{\prime \prime} \mathrm{N} \\
108^{\circ} 29^{\prime} 3 " \mathrm{O}\end{array}$ & 47 & $\begin{array}{l}\text { 29-jun-14- } \\
\text { jul-2019 }\end{array}$ & $\begin{array}{l}\text { Orozco-Gil, } \\
\text { M. }\end{array}$ & CZUG & $*$ \\
\hline $\begin{array}{l}\text { Forelius } \\
\text { mccooki } \\
(\text { McCook, 1880) }\end{array}$ & Sinaloa & & & & & & $\begin{array}{l}\text { Rojas-Fernández, } \\
1996\end{array}$ \\
\hline $\begin{array}{l}\text { Forelius } \\
\text { pruinosus } \\
\text { (Roger, 1863) } \\
\end{array}$ & Ahome & $\begin{array}{l}25^{\circ} 47^{\prime} 25.677^{\prime \prime} \mathrm{N} \\
108^{\circ} 59^{\prime} 9.189^{\prime \prime} \mathrm{O}\end{array}$ & 15 & 18 -oct-74 & Gregg, R. E. & & AntWeb, 2018 \\
\hline \multicolumn{8}{|c|}{ TRIBU: Tapinomini Emery, 1913} \\
\hline $\begin{array}{l}\text { Liometopum } \\
\text { apiculatum } \\
\text { Mayr, } 1870 \\
\end{array}$ & Culiacán & & & 21-ago-64 & Schlinger, R. & & $\begin{array}{l}\text { Del Toro et al. } \\
2009\end{array}$ \\
\hline $\begin{array}{l}\text { Tapinoma } \\
\text { melanocephalum } \\
\text { (Fabricius, 1793) }\end{array}$ & Mazatlán & $\begin{array}{l}23^{\circ} 14^{\prime} 9.996^{\prime \prime} \mathrm{N}, \\
106^{\circ} 24^{\prime} 54.9936^{\prime \prime} \\
\mathrm{O}\end{array}$ & 0 & 20 -oct-74 & Gregg, R. E. & & AntWeb, 2018 \\
\hline $\begin{array}{l}\text { Tapinoma } \\
\text { melanocephalum }\end{array}$ & Ahome & $\begin{array}{l}25^{\circ} 55^{\prime} 10.6^{\prime \prime} \mathrm{N} \\
109^{\circ} 10^{\prime} 27.2^{\prime \prime} \mathrm{O}\end{array}$ & 10 & 18-ago-14 & $\begin{array}{l}\text { Rosas-Mejía, } \\
\text { M. }\end{array}$ & MIEA & $*$ \\
\hline $\begin{array}{l}\text { Tapinoma } \\
\text { melanocephalum }\end{array}$ & Ahome & $\begin{array}{l}25^{\circ} 46^{\prime} 55^{\prime \prime} \mathrm{N} \\
108^{\circ} 59^{\prime} 12^{\prime \prime} \mathrm{O} \\
\end{array}$ & 16 & 04-abr-15 & $\begin{array}{l}\text { Vásquez- } \\
\text { Bolaños, M. }\end{array}$ & CZUG & $*$ \\
\hline $\begin{array}{l}\text { Tapinoma } \\
\text { melanocephalum }\end{array}$ & Ahome & $\begin{array}{l}25^{\circ} 466^{\prime} 55^{\prime \prime} \mathrm{N} \\
108^{\circ} 59^{\prime} 12^{\prime \prime} \mathrm{O}\end{array}$ & 16 & 05-ago-16 & $\begin{array}{l}\text { Vásquez- } \\
\text { Bolaños, M. }\end{array}$ & CZUG & $*$ \\
\hline \multicolumn{8}{|c|}{$\begin{array}{l}\text { SUBFAMILIA FORMICINAE Latreille, } 1809 \\
\text { TRIBU: Camponotini Forel, } 1878\end{array}$} \\
\hline $\begin{array}{l}\text { Camponotus } \\
\text { atriceps (Smith, } \\
1858 \text { ) }\end{array}$ & Ahome & $\begin{array}{l}25^{\circ} 47^{\prime} 23 " \mathrm{~N} \\
109^{\circ} 0^{\prime} 1 " \mathrm{O}\end{array}$ & 13 & 06-ago-10 & $\begin{array}{l}\text { Vásquez- } \\
\text { Bolaños, M. }\end{array}$ & CZUG & $*$ \\
\hline
\end{tabular}




\begin{tabular}{|c|c|c|c|c|c|c|c|}
\hline Especie & Municipio & Coordenadas & $\begin{array}{l}\text { Altitud } \\
(\mathrm{msnm})\end{array}$ & $\begin{array}{c}\text { Fecha de } \\
\text { colecta }\end{array}$ & Colectores & Colección & $\begin{array}{c}\text { Fuente de } \\
\text { información }\end{array}$ \\
\hline $\begin{array}{l}\text { Camponotus } \\
\text { atriceps }\end{array}$ & Guasave & $\begin{array}{l}25^{\circ} 39^{\prime} 27.4^{\prime \prime} \mathrm{N}, \\
108^{\circ} 38^{\prime} 04.8^{\prime \prime} \mathrm{O}\end{array}$ & 15 & 08-jul-14 & $\begin{array}{l}\text { Rosas-Mejía, } \\
\text { M. }\end{array}$ & MIEA & $*$ \\
\hline $\begin{array}{l}\text { Camponotus } \\
\text { atriceps }\end{array}$ & Guasave & $\begin{array}{l}25^{\circ} 34^{\prime} 5^{\prime \prime} \mathrm{N} \\
108^{\circ} 29^{\prime} 3 " \mathrm{O}\end{array}$ & 47 & $\begin{array}{l}\text { 29-jun- } \\
\text { 14.jul.2019 }\end{array}$ & $\begin{array}{l}\text { Orozco-Gil, } \\
\text { M. }\end{array}$ & CZUG & $*$ \\
\hline $\begin{array}{l}\text { Camponotus } \\
\text { consipcuus } \\
\text { (Smith, 1858) } \\
\end{array}$ & Guasave & $\begin{array}{l}25^{\circ} 34^{\prime} 36.9984^{\prime \prime N}, \\
108^{\circ} 27^{\prime} 17.9994^{\prime \prime} \\
\mathrm{O}\end{array}$ & 64 & & $\begin{array}{l}\text { Mackay, W. } \\
\text { P. }\end{array}$ & MWP & $\begin{array}{l}\text { Guénard et al. } \\
2017\end{array}$ \\
\hline $\begin{array}{l}\text { Camponotus } \\
\text { fumidus Roger, } \\
1863\end{array}$ & & & 1609 & & & & $\begin{array}{l}\text { Guénard et al. } \\
2017\end{array}$ \\
\hline $\begin{array}{l}\text { Camponotus } \\
\text { mina Forel, } 1879\end{array}$ & Mazatlán & $\begin{array}{l}23^{\circ} 14^{\prime} 9.996^{\prime \prime} \mathrm{N}, \\
106^{\circ} 24^{\prime} 54.9936^{\prime \prime} \mathrm{O}\end{array}$ & & 20 -oct-74 & Gregg, E. V. & FMNH & AntWeb, 2018 \\
\hline $\begin{array}{l}\text { Camponotus } \\
\text { pullatus Mayr, } \\
1866\end{array}$ & Sinaloa & & & & & & $\begin{array}{l}\text { Vásquez-Bolaños, } \\
2011\end{array}$ \\
\hline $\begin{array}{l}\text { Camponotus } \\
\text { rectangularis } \\
\text { Emery, } 1890 \\
\end{array}$ & Sinaloa & & & & & & $\begin{array}{l}\text { Guénard et al. } \\
2017\end{array}$ \\
\hline $\begin{array}{l}\text { Colobopsis } \\
\text { cerberula } \\
\text { Emery, } 1920\end{array}$ & Mazatlán & $\begin{array}{l}23^{\circ} 14^{\prime} 9.996^{\prime \prime} \mathrm{N}, \\
106^{\circ} 0^{\prime} 0^{\prime \prime} \mathrm{O}\end{array}$ & & 20-oct-74 & $\begin{array}{l}\text { Gregg, R. E. } \\
\text { y Gregg, E. } \\
\text { V. }\end{array}$ & FMNH & $\begin{array}{l}\text { Guénard et al. } \\
2017\end{array}$ \\
\hline $\begin{array}{l}\text { Colobopsis } \\
\text { mathildeae } \\
\text { (Smith, 1949) } \\
\end{array}$ & Concordia & & & 21-oct-74 & Gregg, R. E. & FMNH & AntWeb, 2018 \\
\hline \multicolumn{8}{|c|}{ TRIBU: Lasiini Ashmead, 1905} \\
\hline $\begin{array}{l}\text { Lasius humilis } \\
\text { Wheeler, } 1917\end{array}$ & Concordia & $\begin{array}{l}23^{\circ} 24^{\prime} 26.2^{\prime \prime} \mathrm{N} \\
105^{\circ} 54^{\prime} 16.2^{\prime \prime} \mathrm{O}\end{array}$ & 821 & 19-may-04 & $\begin{array}{l}\text { Camarena, C. } \\
\text { y Vásquez, } \\
\text { M. col. }\end{array}$ & CZUG & $*$ \\
\hline $\begin{array}{l}\text { Nylanderia } \\
\text { terricola } \\
\text { (Buckley, 1866) }\end{array}$ & Mazatlán & $\begin{array}{l}23^{\circ} 14^{\prime} 9.996^{\prime \prime} \\
\mathrm{N}, 106^{\circ} 24^{\prime} 54.993 " \\
\mathrm{O}\end{array}$ & & 20 -oct-74 & Gregg, R. E. & FMNH & AntWeb, 2018 \\
\hline $\begin{array}{l}\text { Paratrechina } \\
\text { longicornis } \\
\text { (Latreille, 1802) }\end{array}$ & Mazatlán & $\begin{array}{l}23^{\circ} 13^{\prime} 0.1194^{\prime \prime} \mathrm{N}, \\
106^{\circ} 25^{\prime} 1.2^{\prime \prime} \mathrm{O}\end{array}$ & & 31-ago-66 & Hamton, R. J. & ANIC & AntWeb, 2018 \\
\hline $\begin{array}{l}\text { Paratrechina } \\
\text { longicornis }\end{array}$ & Guasave & $\begin{array}{l}23^{\circ} 13^{\prime} 0.12^{\prime \prime} \mathrm{N}, \\
106^{\circ} 25^{\prime} 1.2^{\prime \prime} \mathrm{O}\end{array}$ & 20 & 14-ago-14 & $\begin{array}{l}\text { Rosas-Mejía, } \\
\text { M. }\end{array}$ & MIEA & $*$ \\
\hline $\begin{array}{l}\text { Paratrechina } \\
\text { longicornis }\end{array}$ & Ahome & $\begin{array}{l}25^{\circ} 47^{\prime} 23^{\prime \prime} \mathrm{N} \\
109^{\circ} 0^{\prime} 1 " \mathrm{O}\end{array}$ & 13 & 06-ago-10 & $\begin{array}{l}\text { Vásquez- } \\
\text { Bolaños, M. }\end{array}$ & CZUG & $*$ \\
\hline $\begin{array}{l}\text { Paratrechina } \\
\text { longicornis }\end{array}$ & Ahome & $\begin{array}{l}25^{\circ} 35^{\prime} 55^{\prime \prime} \mathrm{N} \\
109^{\circ} 2 ' 57^{\prime \prime} \mathrm{O}\end{array}$ & 2 & $05-a b r-15$ & $\begin{array}{l}\text { Vásquez- } \\
\text { Bolaños, M. }\end{array}$ & CZUG & $*$ \\
\hline $\begin{array}{l}\text { Paratrechina } \\
\text { longicornis }\end{array}$ & El Fuerte & $\begin{array}{l}26^{\circ} 25^{\prime} 19^{\prime \prime} \mathrm{N}, \\
108^{\circ} 37^{\prime} 15^{\prime \prime} \mathrm{O}\end{array}$ & 86 & $05-a b r-15$ & $\begin{array}{l}\text { Vásquez- } \\
\text { Bolaños, M. }\end{array}$ & CZUG & * \\
\hline $\begin{array}{l}\text { Paratrechina } \\
\text { longicornis }\end{array}$ & Ahome & $\begin{array}{l}25^{\circ} 466^{\prime} 55^{\prime \prime} \mathrm{N}, \\
108^{\circ} 59^{\prime} 12^{\prime \prime} \mathrm{O}\end{array}$ & 16 & 05-ago-16 & $\begin{array}{l}\text { Vásquez- } \\
\text { Bolaños, M. }\end{array}$ & CZUG & $*$ \\
\hline $\begin{array}{l}\text { Paratrechina } \\
\text { longicornis }\end{array}$ & Mazatlán & $\begin{array}{l}23^{\circ} 13^{\prime} 46^{\prime \prime} \mathrm{N}, \\
106^{\circ} 25^{\prime} 40^{\prime \prime} \mathrm{O}\end{array}$ & 13 & 05-ago-16 & $\begin{array}{l}\text { Vásquez- } \\
\text { Bolaños, M. }\end{array}$ & CZUG & $*$ \\
\hline $\begin{array}{l}\text { Prenolepis } \\
\text { imparis (Say, } \\
1836 \text { ) }\end{array}$ & Rosario & & & 17-ago-72 & $\begin{array}{l}\text { Gregg, R. E. } \\
\text { y Gregg, E. } \\
\text { V. }\end{array}$ & FMNH & $\begin{array}{l}\text { Guénard et al. } \\
2017\end{array}$ \\
\hline \multicolumn{8}{|c|}{ TRIBU: Myrmelachistini Forel, 1912} \\
\hline $\begin{array}{l}\text { Brachymyrmex } \\
\text { depilis Emery, } \\
1893\end{array}$ & Ahome & $\begin{array}{l}25^{\circ} 55^{\prime} 10.6^{\prime \prime} \mathrm{N} \\
109^{\circ} 10^{\prime} 27.2^{\prime \prime} \mathrm{O}\end{array}$ & 10 & 02-mar-15 & $\begin{array}{l}\text { Rosas-Mejía, } \\
\text { M. }\end{array}$ & MIEA & $*$ \\
\hline
\end{tabular}




\begin{tabular}{|c|c|c|c|c|c|c|}
\hline Especie & Municipio & Coordenadas & $\begin{array}{l}\text { Fecha de } \\
\text { colecta }\end{array}$ & Colectores & Colección & $\begin{array}{l}\text { Fuente de } \\
\text { información }\end{array}$ \\
\hline $\begin{array}{l}\text { Brachymyrmex } \\
\text { heeri Forel, } 1874\end{array}$ & Mazatlán & $\begin{array}{l}23^{\circ} 14^{\prime} 9.996^{\prime \prime} \mathrm{N} \\
106^{\circ} 24^{\prime} 54.9936^{\prime \prime} \mathrm{O}\end{array}$ & 16-ago-72 & Gregg, R. E. & & AntWeb, 2018 \\
\hline \multicolumn{7}{|c|}{ TRIBU: Plagiolepidini Forel, 1886} \\
\hline $\begin{array}{l}\text { Anoplolepis } \\
\text { gracilipes } \\
\text { (Smith, 1857) } \\
\end{array}$ & Sinaloa & & & & & Kempf 1972 \\
\hline $\begin{array}{l}\text { Anoplolepis } \\
\text { gracilipes }\end{array}$ & Mazatlán & $\begin{array}{l}23^{\circ} 14^{\prime} 28^{\prime \prime} \mathrm{N} \\
106^{\circ} 266^{\prime} 55^{\prime \prime} \mathrm{O}\end{array}$ & 17-may-04 & $\begin{array}{l}\text { Camarena- } \\
\text { Bernard, C. y } \\
\text { Vásquez- } \\
\text { Bolaños, M. }\end{array}$ & CZUG & $*$ \\
\hline \multicolumn{7}{|c|}{$\begin{array}{l}\text { PSEUDOMYRMECINAE Smith, } 1952 \\
\text { TRIBU: Pseudomyrmecini Smith, } 1952\end{array}$} \\
\hline $\begin{array}{l}\text { Pseudomyrmex } \\
\text { apache } \\
\text { Creighton, } 1953\end{array}$ & Rosario & & & & SDNMH & AntWeb, 2018 \\
\hline $\begin{array}{l}\text { Pseudomyrmex } \\
\text { championi } \\
\text { (Forel, 1899) }\end{array}$ & Concordia & $\begin{array}{l}23^{\circ} 34^{\prime} 35.1114^{\prime \prime} \mathrm{N}, \\
105^{\circ} 49^{\prime} 57.2874^{\prime \prime} \mathrm{O}\end{array}$ & 15 -ene-07 & & & AntWeb, 2018 \\
\hline $\begin{array}{l}\text { Pseudomyrmex } \\
\text { cubaensis (Forel, } \\
\text { 1901) }\end{array}$ & Mazatlán & & & $\begin{array}{l}\text { Schlinger, E. } \\
\text { I. }\end{array}$ & & Ward, 1989 \\
\hline $\begin{array}{l}\text { Pseudomyrmex } \\
\text { ejectus (Smith, } \\
\text { 1858) }\end{array}$ & Mazatlán & $\begin{array}{l}23^{\circ} 15^{\prime} 0^{\prime \prime} \mathrm{N} \\
106^{\circ} 12^{\prime} 0^{\prime \prime} \mathrm{O}\end{array}$ & 26-may-25 & Keifer, H. H. & & AntWeb, 2018 \\
\hline $\begin{array}{l}\text { Pseudomyrmex } \\
\text { elongatus (Mayr, } \\
\text { 1870) }\end{array}$ & Mazatlán & $\begin{array}{l}23^{\circ} 14^{\prime} 9.996^{\prime \prime} \mathrm{N} \\
106^{\circ} 24^{\prime} 54.9936^{\prime \prime} \mathrm{O}\end{array}$ & 20-oct-74 & Gregg, R. E. & FMNH & AntWeb, 2018 \\
\hline $\begin{array}{l}\text { Pseudomyrmex } \\
\text { hesperius Ward, } \\
1993\end{array}$ & Concordia & $\begin{array}{l}23^{\circ} 23^{\prime} 59.9994^{\prime \prime} \mathrm{N} \\
105^{\circ} 55^{\prime} 59.9874^{\prime \prime} \mathrm{O}\end{array}$ & 09-jun-67 & Janzen, D. H. & PSWC & AntWeb, 2018 \\
\hline $\begin{array}{l}\text { Pseudomyrmex } \\
\text { janzeni Ward, } \\
1993\end{array}$ & Mazatlán & $\begin{array}{l}23^{\circ} 19^{\prime} 0.0114^{\prime \prime} \mathrm{N} \\
106^{\circ} 0^{\prime} 0^{\prime \prime} \mathrm{O}\end{array}$ & 01-ago-64 & $\begin{array}{l}\text { Schlinger, E. } \\
\text { I. }\end{array}$ & PSWC & AntWeb, 2018 \\
\hline $\begin{array}{l}\text { Pseudomyrmex } \\
\text { major (Forel, } \\
\text { 1899) }\end{array}$ & Mazatlán & $\begin{array}{l}23^{\circ} 13^{\prime} 59.9874^{\prime \prime} \mathrm{N} \\
106^{\circ} 25^{\prime} 0.0114^{\prime \prime} \mathrm{O}\end{array}$ & 27-dic-68 & Briggs, D. L. & & AntWeb, 2018 \\
\hline $\begin{array}{l}\text { Pseudomyrmex } \\
\text { pallidus (Smith, } \\
\text { 1855) }\end{array}$ & Mazatlán & $\begin{array}{l}23^{\circ} 31^{\prime} 59.9874 " \mathrm{~N} \\
106^{\circ} 28^{\prime} 59.9874 " \mathrm{O}\end{array}$ & & Siri, M. L. & & AntWeb, 2018 \\
\hline $\begin{array}{l}\text { Pseudomyrmex } \\
\text { seminole Ward, } \\
1985\end{array}$ & Guasave & $\begin{array}{l}25^{\circ} 39^{\prime} 46^{\prime \prime} \mathrm{N} \\
108^{\circ} 30^{\prime} 33^{\prime \prime} \mathrm{O}\end{array}$ & 20-jul-19 & $\begin{array}{l}\text { Rosas-Mejía, } \\
\text { M. }\end{array}$ & MIEA & $*$ \\
\hline $\begin{array}{l}\text { Pseudomyrmex } \\
\text { veneficus } \\
\text { (Wheeler, 1942) }\end{array}$ & Mazatlán & $\begin{array}{l}23^{\circ} 10^{\prime} 0.0114^{\prime \prime} \mathrm{N} \\
106^{\circ} 12^{\prime} 0^{\prime \prime} \mathrm{O}\end{array}$ & 14-sep-63 & Janzen, D. H. & PSWC & AntWeb, 2018 \\
\hline $\begin{array}{l}\text { Pseudomyrmex } \\
\text { veneficus }\end{array}$ & Escuinapa & & & $\begin{array}{l}\text { De Mauzo, L. } \\
\text { y Batty, J. H. }\end{array}$ & & Ward, 1993 \\
\hline \multicolumn{7}{|c|}{ DORYLINAE Leach, 1815} \\
\hline $\begin{array}{l}\text { Eciton burchellii } \\
\text { (Westwood, } \\
\text { 1842) }\end{array}$ & Concordia & $\begin{array}{l}23^{\circ} 16^{\prime} 59.9982^{\prime \prime} \mathrm{N}, \\
106^{\circ} 3^{\prime} 59.997 " \mathrm{O}\end{array}$ & 24-jul-87 & $\begin{array}{l}\text { Bloomfield, } \\
\text { N. }\end{array}$ & SDNHM & AntWeb, 2018 \\
\hline
\end{tabular}




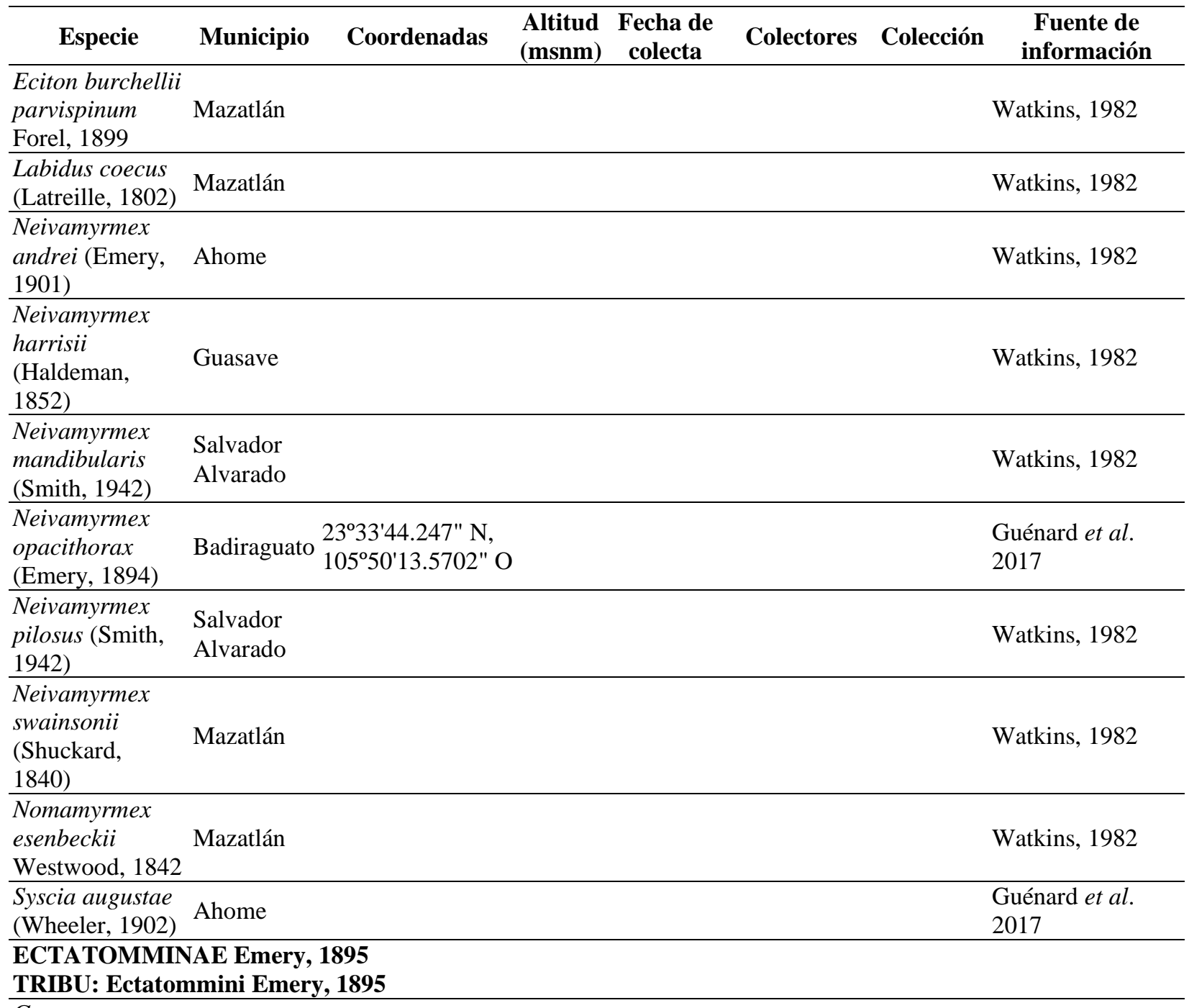

Gnamptogenys

striatula Mayr, Mazatlán

Lattke, 1995

1884

PONERINAE Lepeletier, 1835

TRIBU: Ponerini Lepeletier, 1835

\begin{tabular}{|c|c|c|c|c|c|c|c|}
\hline $\begin{array}{l}\text { Hypoponera } \\
\text { opaciceps } \\
\text { (Mayr, 1887). } \\
\end{array}$ & Ahome & $\begin{array}{l}25^{\circ} 45^{\prime} 23.7^{\prime \prime} \mathrm{N}, \\
108^{\circ} 49^{\prime} 13.0^{\prime \prime} \mathrm{O}\end{array}$ & 12 & 05-may-14 & $\begin{array}{l}\text { Rosas-Mejía, } \\
\text { M. }\end{array}$ & MIEA & $*$ \\
\hline $\begin{array}{l}\text { Neoponera } \\
\text { villosa } \\
\text { (Fabricius, 1804) }\end{array}$ & Sinaloa & & & & & & Forel, 1899 \\
\hline $\begin{array}{l}\text { Odontomachus } \\
\text { clarus Roger, } \\
1861\end{array}$ & Mazatlán & $\begin{array}{l}23^{\circ} 14^{\prime} 9.996^{\prime \prime} \\
\mathrm{N}, 106^{\circ} 24^{\prime} 54.9936^{\prime \prime} \\
\mathrm{O}\end{array}$ & & 16-ago-72 & $\begin{array}{l}\text { Gregg, R. E. } \\
\text { y Gregg, E. } \\
\text { V. }\end{array}$ & FMNH & AntWeb, 2018 \\
\hline $\begin{array}{l}\text { Odontomachus } \\
\text { clarus }\end{array}$ & Guasave & $\begin{array}{l}25^{\circ} 39^{\prime} 27.4^{\prime \prime} \mathrm{N}, \\
108^{\circ} 38^{\prime} 04.8^{\prime \prime} \mathrm{O}\end{array}$ & 15 & 14-ago-14 & $\begin{array}{l}\text { Rosas-Mejía, } \\
\text { M. }\end{array}$ & MIEA & $*$ \\
\hline $\begin{array}{l}\text { Odontomachus } \\
\text { clarus }\end{array}$ & Guasave & $\begin{array}{l}25^{\circ} 34^{\prime} 5^{\prime \prime} \mathrm{N} \\
108^{\circ} 29^{\prime} 3^{\prime \prime} \mathrm{O}\end{array}$ & 47 & $\begin{array}{l}\text { 29-jun-14- } \\
\text { jul-2019 }\end{array}$ & $\begin{array}{l}\text { Orozco-Gil, } \\
\text { M. }\end{array}$ & CZUG & $*$ \\
\hline $\begin{array}{l}\text { Odontomachus } \\
\text { haematodus } \\
\text { (Linnaeus, 1758) }\end{array}$ & Mazatlán & & & & & & $\begin{array}{l}\text { Guénard et al. } \\
2017\end{array}$ \\
\hline
\end{tabular}




\begin{tabular}{|c|c|c|c|c|c|c|c|}
\hline Especie & Municipio & Coordenadas & $\begin{array}{l}\text { Altitud } \\
(\mathbf{m s n m})\end{array}$ & $\begin{array}{c}\text { Fecha de } \\
\text { colecta }\end{array}$ & Colectores & Colección & $\begin{array}{l}\text { Fuente de } \\
\text { información }\end{array}$ \\
\hline $\begin{array}{l}\text { Odontomachus } \\
\text { laticeps Roger, } \\
1861\end{array}$ & Ahome & $\begin{array}{l}23^{\circ} 47^{\prime} 46.6074 " \mathrm{~N} \\
106^{\circ} 51^{\prime} 58.8594^{\prime \prime} \mathrm{O}\end{array}$ & & 17-ago-85 & Ekis, G. & & $\begin{array}{l}\text { Landero-Torres et } \\
\text { al. } 2015\end{array}$ \\
\hline \multicolumn{8}{|c|}{$\begin{array}{l}\text { MYRMICINAE Lepeletier, } 1835 \\
\text { TRIBU: Attini Smith, } 1858\end{array}$} \\
\hline $\begin{array}{l}\text { Acromyrmex } \\
\text { versicolor } \\
\text { (Pergande, 1894) }\end{array}$ & El Fuerte & $\begin{array}{l}26^{\circ} 10^{\prime} 16.269^{\prime \prime} \mathrm{N} \\
108^{\circ} 45^{\prime} 53.4954^{\prime \prime} \mathrm{O}\end{array}$ & & 28-jul-38 & $\begin{array}{l}\text { Michelbacher, } \\
\text { A. E. y Ross, } \\
\text { E. S. }\end{array}$ & CASC & AntWeb, 2018 \\
\hline $\begin{array}{l}\text { Atta cephalotes } \\
\text { (Linnaeus, } 1758 \text { ) }\end{array}$ & Sinaloa & & & & & & $\begin{array}{l}\text { Padilla \& } \\
\text { Miyazawa, } 1972\end{array}$ \\
\hline $\begin{array}{l}\text { Atta mexicana } \\
\text { (Smith, 1858) }\end{array}$ & Mazatlán & $\begin{array}{l}23^{\circ} 11^{\prime} 35.26^{\prime \prime} \mathrm{N}, \\
106^{\circ} 25^{\prime} 16.87^{\prime \prime} \mathrm{O}\end{array}$ & 10 & 17-jun-16 & $\begin{array}{l}\text { Ramírez- } \\
\text { Santana, B. P. }\end{array}$ & CZUG & $*$ \\
\hline Atta mexicana & Guasave & $\begin{array}{l}25^{\circ} 34^{\prime} 5 " \mathrm{~N} \\
108^{\circ} 29^{\prime} 3 " \mathrm{O}\end{array}$ & 47 & $\begin{array}{l}\text { 29-jun- } \\
\text { 14.jul.2019 }\end{array}$ & $\begin{array}{l}\text { Orozco-Gil, } \\
\text { M. }\end{array}$ & CZUG & $*$ \\
\hline $\begin{array}{l}\text { Cephalotes } \\
\text { hirsutus De } \\
\text { Andrade, } 1999 \\
\end{array}$ & Concordia & & & & $\begin{array}{l}\text { Schlinger, E. } \\
\text { I. }\end{array}$ & & $\begin{array}{l}\text { De Andrade \& } \\
\text { Baroni-Urbani, } \\
1999\end{array}$ \\
\hline $\begin{array}{l}\text { Cephalotes } \\
\text { insularis } \\
\text { (Wheeler, 1934) }\end{array}$ & Mazatlán & & & & & & Snelling, 1968 \\
\hline $\begin{array}{l}\text { Cephalotes } \\
\text { rohweri } \\
\text { (Wheeler, 1916) }\end{array}$ & Ahome & & & $10-\operatorname{mar}-69$ & $\begin{array}{l}\text { Snelling, R. } \\
\text { R. }\end{array}$ & & $\begin{array}{l}\text { De Andrade \& } \\
\text { Baroni Urbani, } \\
1999\end{array}$ \\
\hline $\begin{array}{l}\text { Cephalotes } \\
\text { scutulatus } \\
\text { (Smith, 1867) }\end{array}$ & Mazatlán & $\begin{array}{l}23^{\circ} 14^{\prime} 9.996^{\prime \prime} \mathrm{N} \\
106^{\circ} 24^{\prime} 54.9936^{\prime \prime} \mathrm{O}\end{array}$ & & 20 -oct-74 & Gregg, E. V. & & AntWeb, 2018 \\
\hline $\begin{array}{l}\text { Cyphomyrmex } \\
\text { flavidus } \\
\text { Pergande, } 1896\end{array}$ & Culiacan & & & 28-ago-59 & $\begin{array}{l}\text { Stange, L. A. } \\
\text { y Menke, A. } \\
\text { S. }\end{array}$ & & $\begin{array}{l}\text { Snelling \& } \\
\text { Longino, } 1992\end{array}$ \\
\hline \multicolumn{8}{|c|}{ TRIBU: Crematogastrini Forel, 1893} \\
\hline $\begin{array}{l}\text { Crematogaster } \\
\text { crinosa Mayr, } \\
1862\end{array}$ & Concordia & & & 22-ago-64 & $\begin{array}{l}\text { Schlinger, E. } \\
\text { I. }\end{array}$ & & Longino, 2003 \\
\hline $\begin{array}{l}\text { Crematogaster } \\
\text { depilis (Wheeler, } \\
\text { 1919) }\end{array}$ & Rosario & & 1609 & & & & $\begin{array}{l}\text { Guénard et al. } \\
2017\end{array}$ \\
\hline $\begin{array}{l}\text { Crematogaster } \\
\text { opaca Mayr, } \\
1870\end{array}$ & Concordia & & & & & & $\begin{array}{l}\text { Morgan \& } \\
\text { Mackay, } 2017\end{array}$ \\
\hline $\begin{array}{l}\text { Crematogaster } \\
\text { rochai Forel, } \\
1903\end{array}$ & Guasave & $\begin{array}{l}25^{\circ} 39^{\prime} 27.4^{\prime \prime} \mathrm{N} \\
108^{\circ} 38^{\prime} 04.8^{\prime \prime} \mathrm{O}\end{array}$ & & 14-ago-14 & $\begin{array}{l}\text { Rosas-Mejía, } \\
\text { M. }\end{array}$ & MIEA & $*$ \\
\hline $\begin{array}{l}\text { Crematogaster } \\
\text { rochai }\end{array}$ & Escuinapa & & & & & & $\begin{array}{l}\text { Morgan \& } \\
\text { Mackay, } 2017 \\
\end{array}$ \\
\hline $\begin{array}{l}\text { Crematogaster } \\
\text { torosa Mayr, } \\
1870\end{array}$ & Culiacán & $\begin{array}{l}24^{\circ} 48^{\prime} 17.4594^{\prime \prime} \mathrm{N}, \\
107^{\circ} 23^{\prime} 7.7994^{\prime \prime} \mathrm{O}\end{array}$ & & $01-e n e-62$ & Pullen, B. & FMNH & AntWeb, 2018 \\
\hline $\begin{array}{l}\text { Crematogaster } \\
\text { torosa }\end{array}$ & Ahome & $\begin{array}{l}25^{\circ} 47^{\prime} 25.677^{\prime \prime} \mathrm{N} \\
108^{\circ} 59^{\prime} 9.189^{\prime \prime} \mathrm{O}\end{array}$ & & 18 -oct-74 & Gregg, R. E. & & AntWeb, 2018 \\
\hline $\begin{array}{l}\text { Crematogaster } \\
\text { torosa }\end{array}$ & Rosario & $\begin{array}{l}23^{\circ} \mathrm{N}, 106^{\circ} 12^{\prime} 0^{\prime \prime} \\
\mathrm{O}\end{array}$ & & $30-$ ene-64 & $\begin{array}{l}\text { Schlinger, E. } \\
\text { I. }\end{array}$ & & Longino, 2003 \\
\hline
\end{tabular}




\begin{tabular}{|c|c|c|c|c|c|c|c|}
\hline Especie & Municipio & Coordenadas & $\begin{array}{l}\text { Altitud } \\
(\mathrm{msnm})\end{array}$ & $\begin{array}{c}\text { Fecha de } \\
\text { colecta }\end{array}$ & Colectores & Colección & $\begin{array}{c}\text { Fuente de } \\
\text { información }\end{array}$ \\
\hline $\begin{array}{l}\text { Nesomyrmex } \\
\text { wilda (Smith, } \\
\text { 1943) }\end{array}$ & Concordia & $\begin{array}{l}23^{\circ} 24^{\prime} 25.9194 " \mathrm{~N}, \\
105^{\circ} 54^{\prime} 16.1994^{\prime \prime} \mathrm{O}\end{array}$ & & 21-oct-74 & Gregg, R. E. & & $\begin{array}{l}\text { Guénard et al. } \\
2017\end{array}$ \\
\hline $\begin{array}{l}\text { Tetramorium } \\
\text { lanuginosum } \\
\text { Mayr, } 1870\end{array}$ & Mazatlán & $\begin{array}{l}23^{\circ} 14^{\prime} 9.996^{\prime \prime} \mathrm{N}, \\
106^{\circ} 24^{\prime} 54.9936^{\prime \prime} \mathrm{O}\end{array}$ & & 20 -oct-74 & Gregg, R. E. & & $\begin{array}{l}\text { Guénard et al. } \\
2017\end{array}$ \\
\hline $\begin{array}{l}\text { Tetramorium } \\
\text { simillimum } \\
\text { (Smith, F., 1851) }\end{array}$ & Mazatlán & $\begin{array}{l}23^{\circ} 14^{\prime} 9.996^{\prime \prime} \mathrm{N}, \\
106^{\circ} 24^{\prime} 54.9936^{\prime \prime} \mathrm{O}\end{array}$ & & 16-ago-72 & $\begin{array}{l}\text { Gregg, R. E. } \\
\text { y Gregg, E. } \\
\text { V. }\end{array}$ & FMNH & AntWeb, 2018 \\
\hline \multicolumn{8}{|c|}{ TRIBU: Pogonomyrmecini } \\
\hline $\begin{array}{l}\text { Pogonomyrmex } \\
\text { barbatus (Smith, } \\
1858 \text { ) }\end{array}$ & Sinaloa & & & & & & $\begin{array}{l}\text { Schmidt \& } \\
\text { Snelling, } 1986\end{array}$ \\
\hline $\begin{array}{l}\text { Pogonomyrmex } \\
\text { barbatus }\end{array}$ & Guasave & $\begin{array}{l}25^{\circ} 45^{\prime} 23.7^{\prime \prime} \mathrm{N} \\
108^{\circ} 49^{\prime} 13.0^{\prime \prime} \mathrm{O}\end{array}$ & 12 & 05 -abr-15 & $\begin{array}{l}\text { Rosas-Mejía, } \\
\text { M. }\end{array}$ & MIEA & * \\
\hline $\begin{array}{l}\text { Pogonomyrmex } \\
\text { bicolor Cole, } \\
1968\end{array}$ & Sinaloa & & & & & & $\begin{array}{l}\text { Mackay et al. } \\
1985\end{array}$ \\
\hline $\begin{array}{l}\text { Pogonomyrmex } \\
\text { californicus } \\
\text { (Buckley, 1867) }\end{array}$ & Ahome & $\begin{array}{l}26^{\circ} 759.33 .22 " \mathrm{~N}, \\
108^{\circ} 57^{\prime} 34.761^{\prime \prime} \mathrm{O}\end{array}$ & & 11 -oct-74 & Gregg, E. V. & FMNH & AntWeb, 2018 \\
\hline $\begin{array}{l}\text { Pogonomyrmex } \\
\text { maricopa } \\
\text { Wheeler, } 1914\end{array}$ & $\begin{array}{l}\text { Salvador } \\
\text { Alvarado }\end{array}$ & $\begin{array}{l}25^{\circ} 27^{\prime} 44.6832 " \mathrm{~N}, \\
108^{\circ} 5^{\prime} 38.688^{\prime \prime} \mathrm{O}\end{array}$ & 15 & 19-oct-74 & Gregg, R. E. & FMNH & AntWeb, 2018 \\
\hline $\begin{array}{l}\text { Pogonomyrmex } \\
\text { maricopa }\end{array}$ & Ahome & $\begin{array}{l}25^{\circ} 47^{\prime} 23 " \mathrm{~N}, \\
109^{\circ} 0^{\prime} 1 " \mathrm{O}\end{array}$ & 13 & 06-ago-10 & $\begin{array}{l}\text { Vásquez- } \\
\text { Bolaños, M. }\end{array}$ & CZUG & * \\
\hline $\begin{array}{l}\text { Pogonomyrmex } \\
\text { maricopa }\end{array}$ & Guasave & $\begin{array}{l}25^{\circ} 34^{\prime} 5^{\prime \prime} \mathrm{N}, \\
108^{\circ} 29^{\prime} 3 " \mathrm{O}\end{array}$ & 47 & $\begin{array}{l}\text { 29-jun-14- } \\
\text { jul-2019 }\end{array}$ & $\begin{array}{l}\text { M. Orozco- } \\
\text { Gil }\end{array}$ & CZUG & $*$ \\
\hline $\begin{array}{l}\text { Pogonomyrmex } \\
\text { rugosus Emery, } \\
1895\end{array}$ & Sinaloa & & & & & & $\begin{array}{l}\text { Mackay et al. } \\
1985\end{array}$ \\
\hline $\begin{array}{l}\text { Pogonomyrmex } \\
\text { tenuispinus } \\
\text { Forel, } 1914\end{array}$ & Sinaloa & & & & & & $\begin{array}{l}\text { Rojas-Fernández, } \\
1996\end{array}$ \\
\hline $\begin{array}{l}\text { Pogonomyrmex } \\
\text { wheeleri Olsen, } \\
1934\end{array}$ & Mazatlán & $\begin{array}{l}22^{\circ} 54^{\prime} 16.1634 " \mathrm{~N}, \\
106^{\circ} 24^{\prime} 22.1754^{\prime \prime} \mathrm{O}\end{array}$ & & 14-ago-61 & Cole, A. C. & RAJC & AntWeb, 2018 \\
\hline $\begin{array}{l}\text { Pogonomyrmex } \\
\text { wheeleri }\end{array}$ & Concordia & & 821 & 19-may-04 & $\begin{array}{l}\text { Vásquez, M. } \\
\text { col. }\end{array}$ & CZUG & * \\
\hline \multicolumn{8}{|c|}{ TRIBU: Pheidolini Emery, 1877} \\
\hline $\begin{array}{l}\text { Pheidole } \\
\text { crassicornis } \\
\text { Emery, } 1895\end{array}$ & Rosario & & 1609 & & & FMNH & $\begin{array}{l}\text { Guénard et al. } \\
2017\end{array}$ \\
\hline $\begin{array}{l}\text { Pheidole } \\
\text { crassicornis }\end{array}$ & Culiacán & & & & & FMNH & $\begin{array}{l}\text { Guénard et al. } \\
2017\end{array}$ \\
\hline $\begin{array}{l}\text { Pheidole } \\
\text { fimbriata Roger, } \\
1863\end{array}$ & Escuinapa & & & & Batty, J. H. & & Smith, 1943 \\
\hline $\begin{array}{l}\text { Pheidole } \\
\text { obtusospinosa } \\
\text { Pergande, } 1896\end{array}$ & Sinaloa & & & & & & $\begin{array}{l}\text { Mackay \& } \\
\text { Mackay, } 2002\end{array}$ \\
\hline $\begin{array}{l}\text { Pheidole rhea } \\
\text { Wheeler, } 1908\end{array}$ & Escuinapa & $\begin{array}{l}31^{\prime} 13.5186 " \mathrm{~N}, \\
105^{\circ} 41^{\prime} 48.1914^{\prime \prime} \mathrm{O}\end{array}$ & & 01 -ene-62 & Pullen, B. & FMNH & AntWeb, 2018 \\
\hline
\end{tabular}




\begin{tabular}{|c|c|c|c|c|c|c|c|}
\hline Especie & Municipio & Coordenadas & $\begin{array}{l}\text { Altitud } \\
(\mathrm{msnm})\end{array}$ & $\begin{array}{c}\text { Fecha de } \\
\text { colecta }\end{array}$ & Colectores & Colección & $\begin{array}{c}\text { Fuente de } \\
\text { información }\end{array}$ \\
\hline Pheidole rhea & $\begin{array}{l}\text { Agua } \\
\text { Caliente }\end{array}$ & $\begin{array}{l}23^{\circ} 0^{\prime} 31.5432^{\prime \prime} \mathrm{N}, \\
105^{\circ} 17^{\prime} 54.456^{\prime \prime} \mathrm{O}\end{array}$ & 122 & $17-m a r-74$ & Clark, W. H. & FMNH & AntWeb, 2018 \\
\hline $\begin{array}{l}\text { Pheidole } \\
\text { setsukoae } \\
\text { Wilson, } 2003\end{array}$ & Sinaloa & & & & & & Wilson, 2003 \\
\hline $\begin{array}{l}\text { Pheidole } \\
\text { tepicana } \\
\text { Pergande, } 1896\end{array}$ & Culiacán & $\begin{array}{l}24^{\circ} 48^{\prime} 17.4594^{\prime \prime} \mathrm{N}, \\
107^{\circ} 23^{\prime} 7.7994^{\prime \prime} \mathrm{O}\end{array}$ & & 01 -ene-62 & & FMNH & AntWeb, 2018 \\
\hline $\begin{array}{l}\text { Pheidole tetra } \\
\text { Creighton, } 1950\end{array}$ & Culiacán & $\begin{array}{l}24^{\circ} 48^{\prime} 17.4594^{\prime \prime} \mathrm{N} \\
107^{\circ} 23^{\prime} 7.7994^{\prime \prime} \mathrm{O}\end{array}$ & & 01 -ene-62 & Pullen, B. & FMNH & AntWeb, 2018 \\
\hline Pheidole tetra & Guasave & $\begin{array}{l}25^{\circ} 45^{\prime} 23.7^{\prime \prime} \mathrm{N}, \\
108^{\circ} 49^{\prime} 13.0^{\prime \prime} \mathrm{O}\end{array}$ & 12 & 04-jul-14 & $\begin{array}{l}\text { Rosas-Mejía, } \\
\text { M. }\end{array}$ & MIEA & $*$ \\
\hline \multicolumn{8}{|c|}{ TRIBU:Solenopsidini } \\
\hline $\begin{array}{l}\text { Monomorium } \\
\text { compressum } \\
\text { Wheeler, } 1914 \\
\end{array}$ & Ahome & & & & & & DuBois, 1986 \\
\hline $\begin{array}{l}\text { Monomorium } \\
\text { cyaneum } \\
\text { Wheeler, W. M., } \\
1914\end{array}$ & Ahome & $\begin{array}{l}25^{\circ} 55^{\prime} 10.6^{\prime \prime} \mathrm{N} \\
109^{\circ} 10^{\prime} 27.2^{\prime \prime} \mathrm{O}\end{array}$ & 10 & 08 -abr-15 & $\begin{array}{l}\text { Rosas-Mejía, } \\
\text { M. }\end{array}$ & MIEA & $*$ \\
\hline $\begin{array}{l}\text { Monomorium } \\
\text { floricola } \\
\text { (Jerdon, 1851) }\end{array}$ & Mazatlán & $\begin{array}{l}23^{\circ} 14^{\prime} 9.996^{\prime \prime} \mathrm{N}, \\
106^{\circ} 24^{\prime} 54.9936^{\prime \prime} \mathrm{O}\end{array}$ & & 20 -oct-74 & Gregg, R. E. & FMNH & AntWeb, 2018 \\
\hline $\begin{array}{l}\text { Monomorium } \\
\text { minimum } \\
\text { (Buckley, 1867) }\end{array}$ & Sinaloa & & & & & & $\begin{array}{l}\text { Rojas-Fernández, } \\
1996\end{array}$ \\
\hline $\begin{array}{l}\text { Monomorium } \\
\text { minimum }\end{array}$ & Ahome & $\begin{array}{l}25^{\circ} 35^{\prime} 55^{\prime \prime} \mathrm{N} \\
109^{\circ} 2^{\prime} 57^{\prime \prime} \mathrm{O}\end{array}$ & 2 & 05 -abr-15 & $\begin{array}{l}\text { Vásquez- } \\
\text { Bolaños, M. }\end{array}$ & CZUG & $*$ \\
\hline $\begin{array}{l}\text { Monomorium } \\
\text { minimum }\end{array}$ & Ahome & $\begin{array}{l}25^{\circ} 46^{\prime} 55^{\prime \prime} \mathrm{N}, \\
108^{\circ} 59^{\prime} 12^{\prime \prime} \mathrm{O}\end{array}$ & 16 & 05-ago-16 & $\begin{array}{l}\text { Vásquez- } \\
\text { Bolaños, M. }\end{array}$ & CZUG & $*$ \\
\hline $\begin{array}{l}\text { Monomorium } \\
\text { pharaonis } \\
\text { (Linnaeus, 1758) }\end{array}$ & Ahome & $\begin{array}{l}25^{\circ} 55^{\prime} 10.6^{\prime \prime} \mathrm{N},- \\
109^{\circ} 10^{\prime} 27.2^{\prime \prime O}\end{array}$ & & 09-abr-15 & $\begin{array}{l}\text { Rosas-Mejía, } \\
\text { M. }\end{array}$ & MIEA & $*$ \\
\hline $\begin{array}{l}\text { Monomorium } \\
\text { pharaonis } \\
\text { (Linnaeus, 1758) }\end{array}$ & Ahome & $\begin{array}{l}25^{\circ} 47^{\prime} 23^{\prime \prime} \mathrm{N} \\
109^{\circ} 0^{\prime} 1 " \mathrm{O}\end{array}$ & 13 & 06-ago-10 & $\begin{array}{l}\text { Vásquez- } \\
\text { Bolaños, M. }\end{array}$ & CZUG & $*$ \\
\hline $\begin{array}{l}\text { Monomorium } \\
\text { viridum Brown, } \\
1943\end{array}$ & Culiacán & $\begin{array}{l}24^{\circ} 48^{\prime} 17.4594^{\prime \prime} \mathrm{N} \\
107^{\circ} 23^{\prime} 7.7994^{\prime \prime} \mathrm{O}\end{array}$ & & 01 -ene-62 & Pullen, B. & FMNH & AntWeb, 2018 \\
\hline $\begin{array}{l}\text { Monomorium } \\
\text { viridum }\end{array}$ & Mazatlán & $\begin{array}{l}23^{\circ} 14^{\prime} 9.996^{\prime \prime} \mathrm{N}, \\
106^{\circ} 24^{\prime} 54.9936^{\prime \prime} \mathrm{O}\end{array}$ & & 16-ago-72 & Gregg, R. E. & FMNH & AntWeb, 2018 \\
\hline $\begin{array}{l}\text { Monomorium } \\
\text { viridum }\end{array}$ & Ahome & $\begin{array}{l}25^{\circ} 47^{\prime} 25.677^{\prime \prime} \mathrm{N}, \\
108^{\circ} 59^{\prime} 9.189^{\prime \prime} \mathrm{O}\end{array}$ & 15 & 18-sep-74 & Gregg, R. E. & & AntWeb, 2018 \\
\hline $\begin{array}{l}\begin{array}{l}\text { Solenopsis } \\
\text { geminata } \\
\text { (Fabricius, 1804) }\end{array} \\
\end{array}$ & Guasave & $\begin{array}{l}25^{\circ} 34^{\prime} 36.9984^{\prime \prime} \mathrm{N}, \\
108^{\circ} 27^{\prime} 17.9994^{\prime \prime} \mathrm{O}\end{array}$ & & & Mackay, W. & & $\begin{array}{l}\text { Guénard et al. } \\
2017\end{array}$ \\
\hline $\begin{array}{l}\text { Solenopsis } \\
\text { geminata }\end{array}$ & Ahome & $\begin{array}{l}25^{\circ} 55^{\prime} 10.6^{\prime \prime} \mathrm{N}, \\
109^{\circ} 10^{\prime} 27.2^{\prime \prime} \mathrm{O}\end{array}$ & 10 & 15 -feb-15 & $\begin{array}{l}\text { Rosas-Mejía, } \\
\text { M. }\end{array}$ & MIEA & $*$ \\
\hline $\begin{array}{l}\text { Solenopsis } \\
\text { geminata }\end{array}$ & Ahome & $\begin{array}{l}25^{\circ} 47^{\prime \prime} 23^{\prime \prime} \mathrm{N}, \\
109^{\circ} 00^{\prime} 1 " \mathrm{O}\end{array}$ & 13 & 06-ago-10 & $\begin{array}{l}\text { Vásquez- } \\
\text { Bolaños M. }\end{array}$ & CZUG & $*$ \\
\hline $\begin{array}{l}\text { Solenopsis } \\
\text { geminata }\end{array}$ & Ahome & $\begin{array}{l}25^{\circ} 35^{\prime} 55^{\prime \prime} \mathrm{N} \\
109^{\circ} 2 ' 57^{\prime \prime} \mathrm{O}\end{array}$ & 2 & 05 -abr-15 & $\begin{array}{l}\text { Vásquez- } \\
\text { Bolaños, M. }\end{array}$ & CZUG & * \\
\hline
\end{tabular}




\begin{tabular}{|c|c|c|c|c|c|c|c|}
\hline Especie & Municipio & Coordenadas & $\begin{array}{l}\text { Altitud } \\
(\mathbf{m s n m})\end{array}$ & $\begin{array}{c}\text { Fecha de } \\
\text { colecta }\end{array}$ & Colectores & Colección & $\begin{array}{c}\text { Fuente de } \\
\text { información }\end{array}$ \\
\hline $\begin{array}{l}\text { Solenopsis } \\
\text { geminata }\end{array}$ & Mazatlán & $\begin{array}{l}23^{\circ} 13^{\prime} 46^{\prime \prime} \mathrm{N}, \\
106^{\circ} 25^{\prime} 40^{\prime \prime} \mathrm{O}\end{array}$ & 13 & 06-ago-16 & $\begin{array}{l}\text { Vásquez- } \\
\text { Bolaños, M. }\end{array}$ & CZUG & $*$ \\
\hline $\begin{array}{l}\text { Solenopsis } \\
\text { geminata }\end{array}$ & Guasave & $\begin{array}{l}25^{\circ} 34^{\prime} 5^{\prime \prime} \mathrm{N}, \\
108^{\circ} 29^{\prime} 3 " \mathrm{O}\end{array}$ & 47 & $\begin{array}{l}\text { 29-jun-14- } \\
\text { jul-2019 }\end{array}$ & $\begin{array}{l}\text { Orozco-Gil, } \\
\text { M. }\end{array}$ & CZUG & $*$ \\
\hline $\begin{array}{l}\text { Solenopsis } \\
\text { xyloni McCook, } \\
1880\end{array}$ & Sinaloa & & & & Mackay, W. & & $\begin{array}{l}\text { Mackay \& } \\
\text { Mackay } 2002\end{array}$ \\
\hline \multicolumn{8}{|c|}{ TRIBU: Stenammini Ashmead, 1905} \\
\hline $\begin{array}{l}\text { Novomessor } \\
\text { albisetosus } \\
\text { Mayr, } 1886 \\
\end{array}$ & Culiacán & $\begin{array}{l}24^{\circ} 10^{\prime} 15.999^{\prime \prime} \mathrm{N}, \\
107^{\circ} 1{ }^{\prime} 1.9992^{\prime \prime} \mathrm{O}\end{array}$ & 64 & & Mackay, W. & & $\begin{array}{l}\text { Guénard et al. } \\
2017\end{array}$ \\
\hline $\begin{array}{l}\text { Novomessor } \\
\text { albisetosus }\end{array}$ & Guasave & $\begin{array}{l}25^{\circ} 45^{\prime} 23.7^{\prime \prime} \mathrm{N}, \\
108^{\circ} 49^{\prime} 13.0^{\prime \prime} \mathrm{O}\end{array}$ & 12 & 09-abr-15 & $\begin{array}{l}\text { Rosas-Mejía, } \\
\text { M. }\end{array}$ & MIEA & $*$ \\
\hline $\begin{array}{l}\text { Stenamma } \\
\text { huachucanum } \\
\text { Smith, } 1957\end{array}$ & Concordia & $\begin{array}{l}23^{\circ} 34^{\prime} 41.8794 " \mathrm{~N}, \\
105^{\circ} 50^{\prime} 5.9994^{\prime \prime} \mathrm{O}\end{array}$ & & 15-ene-07 & Ward, P. S. & & Branstetter, 2013 \\
\hline
\end{tabular}

